\title{
Reproducing Space Plasma in the Lab
}

\author{
Electromagnetic fields rotate a plasma and produce conditions that \\ resemble the region around a newly forming star.
}

\section{By Michael Schirber}

A star is born when enough plasma (ionized gas) in a rotating disk spirals into the central, higher-density region. A new experiment could help astrophysicists understand this in-fall, as well as the behavior of magnetic fields in the moving plasma. Researchers induced a rotating flow in a ten-thousand-degree-kelvin plasma using electromagnetic fields to "stir" the ions [1]. They showed that the motion mimics the region around an accreting protostar and observed that the rotation induced an unexpected, 20 -fold amplification of an applied magnetic field. In future

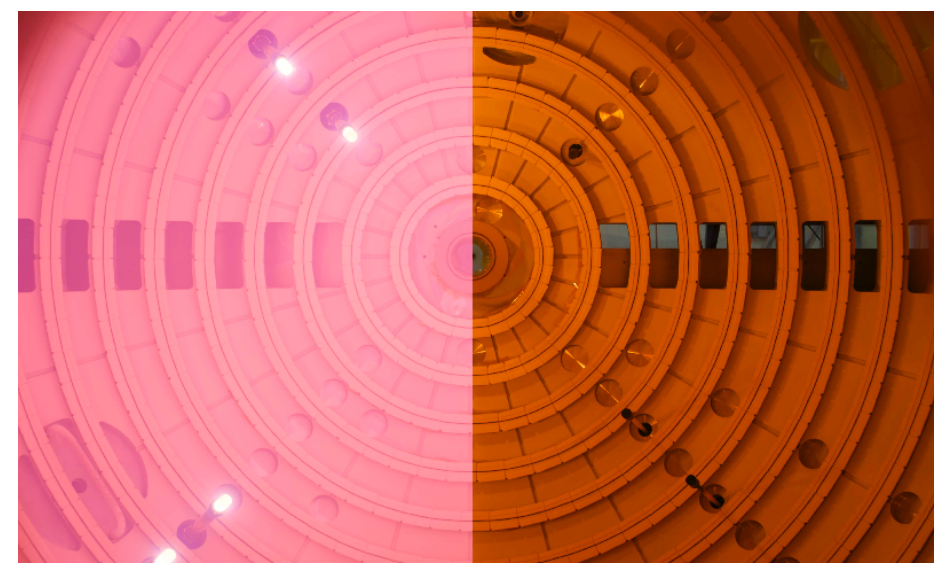

Plasma view. This image pair shows the inside of a spherical plasma vessel. On the left, the vessel is filled with plasma, which is visible as a soft haze. The glowing bulb-like objects are hot cathodes that emit electrons that ionize the gas. On the right, the vessel is empty, allowing a clearer view of the permanent magnets (concentric rings) that confine the plasma with strong magnetic fields.

Credit: K. Flanagan/Univ. of Wisconsin experiments, the team hopes to study the mechanics of the rotation and other aspects of accretion disks.

A protostar grows in size by accreting matter from a rotating disk that surrounds it. This disk contains hot plasma that gradually slows down and falls inward under gravity. One theory explaining how the system can conserve angular momentum during this collapse is that a specific instability-the so-called magnetorotational instability-creates turbulent conditions that cause some material to spiral out and other material to spiral in [2].

Testing this instability theory in the lab with an accretion-disk analog requires rotating a plasma in the so-called Hall regime. In this state, the magnetic field is weak enough and particle collisions are frequent enough that the ions do not move strictly along magnetic field lines. To work in this regime, Ken Flanagan from the University of Wisconsin-Madison and his colleagues have been developing systems where the magnetic field is strong at the boundaries (for containment purposes) but is relatively weak in the center. "We're trying to recreate plasmas in our lab that we can use to test out astrophysical concepts," Flanagan says.

Flanagan and colleagues managed to create stable, rotating plasma in the Hall regime using two separate systems: a 1-m-wide cylindrical vessel and a 3-m-wide spherical vessel. Electrodes ionized the argon gas in each vessel, creating plasma with a temperature of roughly $10,000 \mathrm{~K}$, while magnetic fields kept this hot matter away from the walls.

Without wall contact, the plasma couldn't be rotated by 


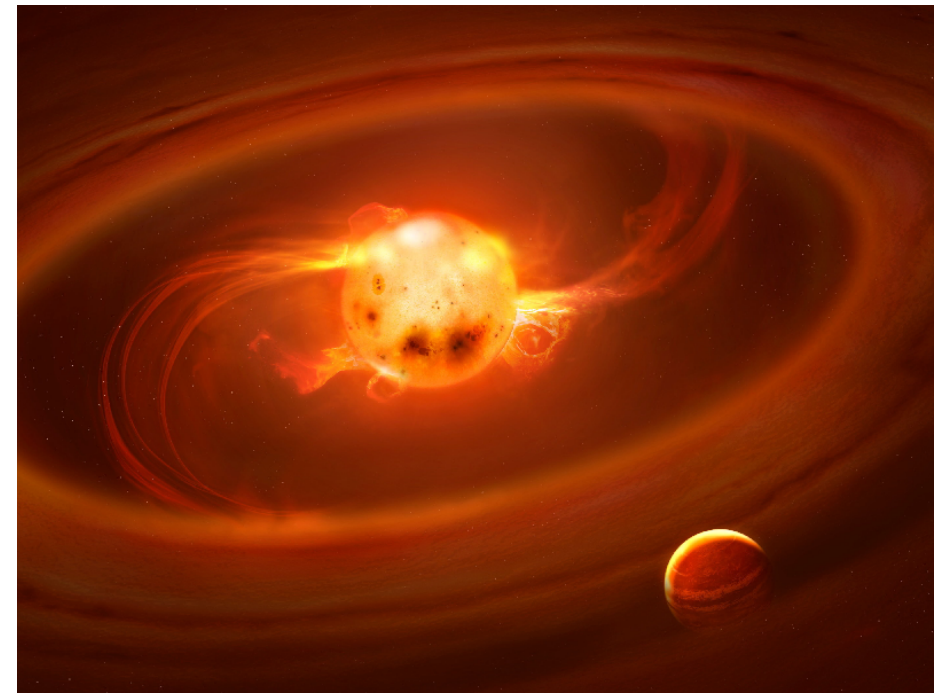

A star is born. In this artist's image, plasma falls in from the disk of material surrounding a growing baby star.

Credit: A. Mark Garlick

spinning the containers. Instead, Flanagan and his colleagues developed an electromagnetic stirring mechanism. A strong electric current is sent through the plasma via electrodes on the walls. In the spherical vessel, the current flows outward from the axis of the cylindrically symmetric magnetic field; in the cylindrical vessel, the current flows inward. In both cases, the combination of current and field creates a torque that rotates the plasma-fastest near the center and slowest around the periphery. This so-called Couette flow is similar to what is expected in accretion disks.

The rotating plasmas do not yet show the instability predicted for accretion flows. But Flanagan believes that the team should be able to tune the parameters-such as the temperature and density of the plasma-in order to induce the instability.

The researchers did, however, witness a different effect that may have astrophysical relevance. When the plasma was stirred with current directed outward, the weak magnetic field that threads through the plasma was amplified by 20 times. By contrast, the magnetic field was reduced dramatically when the current pointed inward. The team believes the effect is caused by the current-carrying electrons taking a spiral path around the central rotation axis. This curling motion generates a magnetic field that either amplifies or cancels the external field, depending on which direction the spiraling goes.

"Magnetic fields are ubiquitous in astrophysics and space physics," says fluid dynamics expert Pablo Mininni from the University of Buenos Aires in Argentina. "But their generation and sustainment by the motion of the plasma in the interstellar or interplanetary medium is still shrouded in mysteries." This experiment shows that a plasma moving in the Hall regime can amplify a preexisting magnetic field, which could help explain the origin of astrophysical magnetic fields, Mininni says.

Astrophysicist Matthew Kunz of Princeton University thinks this work is a significant contribution to our understanding of accretion disks, for which recent theoretical work has shown the importance of effects related to the Hall regime [3]. "The physics involved, while grounded in classical electromagnetism and fluid dynamics, is surprisingly rich and complex, and so it is worth celebrating this new laboratory frontier on its own merits," Kunz says.

Michael Schirber is a Corresponding Editor for Physics based in Lyon, France.

\section{REFERENCES}

1. K. Flanagan et al., "Weakly magnetized, Hall dominated plasma Couette flow," Phys. Rev. Lett. 125, 135001 (2020).

2. S. A. Balbus and J. F. Hawley, "A powerful local shear instability in weakly magnetized disks. I - Linear analysis," Astrophys. J. 376, 214 (1991).

3. M. W. Kunz and G. Lesur, "Magnetic self-organization in Hall-dominated magnetorotational turbulence," Mon. Not. R. Astron. Soc. 434, 2295 (2013). 